\title{
MiR-106b promotes cell proliferation via targeting RB in laryngeal carcinoma
}

\author{
Kemin Cai ${ }^{\dagger}$, Yu Wang ${ }^{\dagger}$ and Xueli Bao*
}

\begin{abstract}
MiR-106b is frequently up-regulated in various types of human cancer including laryngeal carcinoma. However the underlying mechanism of miR-106b involved in laryngeal carcinoma remains elusive. Here we showed that reduction of miR-106b induced cell cycle G0/G1 arrest by targeting tumor suppressor RB in human laryngeal carcinoma cells. Further, Introducing RB cDNA without 3'UTR abrogated miR-106b-induced cell proliferation. Finally, there was an inverse relationship between RB and miR-106b expression in laryngeal carcinoma tissues. To our knowledge, these data indicate for the first time that miR-106b directly regulate cell cycle by targeting RB in laryngeal carcinoma and that miR-106b could be potential therapeutic approaches for laryngeal carcinoma.
\end{abstract}

Keywords: laryngeal carcinoma, miR-106b, RB, cell proliferation

\section{Background}

Laryngeal carcinoma is a common head and neck malignancy with high incidence as it accounts for approximately $2.4 \%$ of new malignancies worldwide every year $[1,2]$. Despite recent advances in cancer treatment, the prognosis for patients with laryngeal carcinoma especially at advanced stage remains poor. Therefore, it is essential to investigate the mechanism involved in the development and progression of laryngeal carcinoma.

MicroRNAs (miRNAs) are a new class of small, noncoding RNAs and regulate gene expression by binding to the 3'-untranslated regions (3'UTRs) of specific mRNAs. miRNAs could function as oncogenic miRNAs or tumor suppressor miRNAs, playing crucial roles in the development and progression of carcer [3,4]. Recent studies have indicated that frequent deregulation of miRNA in laryngeal carcinoma $[5,6]$. Let-7a was significantly downregulated both in human laryngeal squamous cancer tissues and Hep-2 cells, and functions as a potential tumor suppressor in human laryngeal cancer [5]. Hui et al investigated the significance of miRNA in patients with locally advanced head and neck squamous cell carcinoma and identified that thirty-eight miRNAs were significantly differentially expressed between

\footnotetext{
* Correspondence: entcaikemin7173@126.com

† Contributed equally

Department of Otorhinolaryngology Head and Neck Surgery, Taizhou People's Hospital, Taizhou 225300, P.R. China
}

malignant versus normal tissues [6]. Of note, upregulation of miR-106b, miR-423, miR-20a, and miR-16 as well as downregulation of miR-10a were newly observed.

In present work, we determined the function of miR106b involved in laryngeal carcinoma. Reduction of miR$106 \mathrm{~b}$ by antisense oligonucleotides inhibited cell proliferation and induced cell cycle G0/G1 arrest in laryngeal carcinoma cells. Moreover, RB was a direct target of miR-106b by luciferase reporter assay. Introduction of RB CDNA without 3'UTR abrogated miR-106b-induced cell proliferation. Finally, there was an inverse correlation of expression of miR-106b and RB in laryngeal carcinoma tissues.

\section{Materials and methods}

\section{Clinical sample collection}

Twenty laryngeal carcinoma tissues used in this study were obtained from Taizhou People's Hospital in China. Specimens were snap-frozen in liquid nitrogen, incuding 10 laryngeal carcinomas with stage I and II, and 10 laryngeal carcinomas with stage III and IV. The collection and use of the patient samples were reviewed and approved by Institutional Ethics Committees, and written informed consent from all patients was appropriately obtained.

\section{Cell culture and transfection}

Hep-2 and TU212 cells were purchased from Chinese Academy of Sciences Cell Bank. Cells were maintained

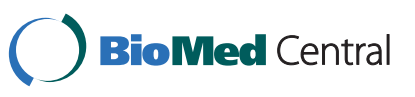

(c) 2011 Cai et al; licensee BioMed Central Ltd. This is an Open Access article distributed under the terms of the Creative Commons Attribution License (http://creativecommons.org/licenses/by/2.0), which permits unrestricted use, distribution, and reproduction in any medium, provided the original work is properly cited. 
in DMEM medium supplemented with $10 \%$ fetal bovine serum. Cells were transfected using Lipofectamine 2000 (Invitrogen, USA) at the time of $50-60 \%$ confluent. $48 \mathrm{~h}$ after transfection, cells were harvested for further studies.

\section{Plasmids and oligonucleotides}

For expression plasmid construct, wild-type RB cDNA sequence without 3'UTR was selected and cloned into Pgenesil-1 vector. 2'-O-methyl (OMe)-oligonucleotides were chemically synthesized and purified by GenePharma Co., Ltd. (Shanghai, China). The amount of oligonucleotides transfected was $50 \mathrm{nmol} / \mathrm{L}$. Sequences as follows: miR-106b, 5'- UAAAGUGCUGACAGUGCAGAU-3'; anti-miR-106b (As-miR-106b), 5'-AUCUGCACUGUCAGCACUUUA-3'; scrambled miRNA (negative control), 5'-UUGUACUACACAAAAGUACUG-3'.

\section{Real time PCR}

Trizol reagent was used to isolate total RNA from cells $48 \mathrm{~h}$ after transfection. The RT-real-time PCR was carried out with the miRNA detection kit (Ambion, USA). Amplification reaction protocol was performed for 40 cycles consisting $95^{\circ} \mathrm{C}$ for $3 \mathrm{~min}, 95^{\circ} \mathrm{C}$ for $15 \mathrm{sec}, 60^{\circ} \mathrm{C}$ for $30 \mathrm{sec}$. Both RT and PCR primer were purchased from Ambion. 5S RNA was used for normalization. Relative quantification was conducted using amplification efficiencies derived from cDNA standard curves and obtained relative gene expression. Relative gene expression was calculated via a $2^{\Delta \Delta \mathrm{Ct}}$ method.

\section{MTT assay}

Cells were plated at $10^{4}$ cells per well in 96-well plates with six replicate wells. After transfection as described previously, $20 \mu \mathrm{l}$ of MTT (5 g/L, Sigma, USA) was added into each well at each day of consecutive 4 days after treatment and the cells were incubated for additional $4 \mathrm{~h}$, the supernatant was then discarded. $200 \mu \mathrm{l}$ of DMSO was added to each well to dissolve the precipitate. Optical density (OD) was measured at wave length of $550 \mathrm{~nm}$. The data are presented as the mean $\pm \mathrm{SD}$, which are derived from triplicate samples of at least three independent experiments.

\section{Cell cycle analysis}

Cells were washed with PBS, fixed with $70 \%$ ethanol for at least $1 \mathrm{~h}$. After extensive washing, the cells were suspended in HBSS (Hank's Balanced Salt Solution) containing $50 \mu \mathrm{g} / \mathrm{mL}$ PI and $50 \mu \mathrm{g} / \mathrm{ml}$ RNase $\mathrm{A}$ and incubated for $1 \mathrm{~h}$ at room temperature, and analyzed by FACScan (Becton Dickinson, USA). Cell cycle analysis was analyzed by ModFit software. Experiments were performed in triplicate. Results were presented as \% of cell in a particular phase.

\section{Western blot analysis}

Equal amounts of protein per lane were separated by $8 \%$ SDS-polyacrylamide gel and transferred to PVDF membrane. The membrane was blocked in 5\% skim milk for $1 \mathrm{~h}$ and then incubated with a specific antibody for $2 \mathrm{~h}$. The antibodies used in this study were: antibodies to RB (Santa Cruz, USA). The antibody against $\beta$-actin (Santa Cruz, USA) was used as control. The specific protein was detected by using a SuperSignal protein detection kit (Pierce, USA). The band density of specific proteins was quantified after normalization with the density of $\beta$ actin.

\section{Luciferase reporter assay}

The human RB 3'UTR (bases 813-959) were amplified and cloned into the XbaI site of the pGL3-control vector (Promega, USA), downstream of the luciferase gene, to generate the plasmids pGL3-WT-RB-3'UTR. pGL3MUT-RB-3'UTR plasmids were generated from pGL3WT-RB-3'UTR by deleting the binding site (bases $883-$ 889) for miR-106b "GCACUUU". For the luciferase reporter assay, cells were cultured in 96-well plates, transfected with the plasmids and As-miR-106b using Lipofectamine 2000. $48 \mathrm{~h}$ after transfection, luciferase activity was measured using the Dual Luciferase Reporter Assay System (Promega). Firefly luciferase activity was normalized to renilla luciferase activity for each transfected well.

\section{Statistical analysis}

Statistics was determined by ANOVA, or $t$ test using SPSS11.0. Statistical significance is determined as $\mathrm{P}<$ 0.05 .

\section{Results}

\section{MiR-106b expression in laryngeal carcinomas}

To explore miR-106b expression in laryngeal carcinomas, we examined 20 human laryngeal carcinoma specimens with different clinical stages using Real time PCR. As shown in Figure 1, the levels of miR-106b increased markedly in laryngeal carcinomas with stage III and IV in comparison to those with stage I and II $(\mathrm{P}<0.01)$. And we also found high miR-106b expression in Hep-2 and TU212 laryngeal carcinoma cells (Figure 1).

\section{MiR-106b inhibition suppresses cell proliferation and induces G0/G1 arrest}

As-miR-106b and miR-106b mimic oligonucleotides were employed to change miR-106b expression in Hep2 and TU212 cells to evaluate the significance of miR106b in laryngeal carcinoma. In both two cells, miR106b expression significantly decreased in As-miR-106b group and increased in miR-106b group $48 \mathrm{~h}$ after transfection (Figure 2A). MTT assay data showed that a 


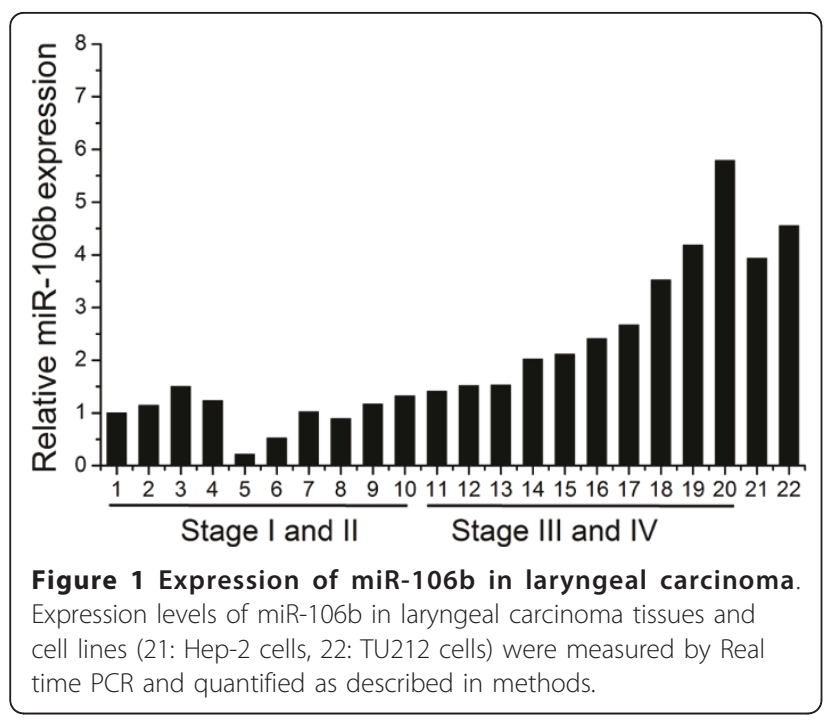

statistically significant cell proliferation inhibition was found in As-miR-106b group of Hep-2 cells, compared with control groups respectively. Similar trend was observed in TU212 cells (Figure 2B). There was no difference between blank control group and negative control group in the whole experiment. Next we analyzed the cell cycle distribution by FACS. As-miR-106b treated cells represented significant ascends in G0/G1 phase in comparison to untreated Hep-2 and TU212 cells
(Figure 2C). However, we did not observe a significant difference in the rate of growth inhibition between miR106b group and blank control group; although a slightly increasing trend of cell survival rate and G0/G1 phase was seen in Hep-2 and TU212 cells. These results raise the possibility that there exists a threshold value for miR-106b up-regulation. Taken together, reduction of miR-106b can induce cells arrest at G0/G1 phases, thereby inhibiting cell proliferation in laryngeal carcinoma cells.

\section{$\mathrm{RB}$ is a direct target of miR-106b}

To further explore the molecular mechanism of As-miR-106b induced cell cycle in laryngeal carcinoma cells, bioinformatics analysis of miR-106b potential target genes was performed through the databases TargetScan http://www.targetscan.org and PicTar http://www. pictar.bio.nyu.edu, We found that tumor suppressor RB associated with cell cycle contained the highly conserved putative miR-106b binding sites (Figure 3A). To determine whether RB is directly regulated by miR-106b, Western blot analysis and Luciferase reporter assay were employed. Western blot analysis showed that a notable induction of RB expression was detected after knockdown of miR-106b in Hep-2 and TU212 cells (Figure 3B). Further, we created pGL3-WT-RB-3'UTR, and pGL3-MUT-RB-3'UTR plasmids. Reporter assay revealed that inhibition of miR-106b triggered a marked

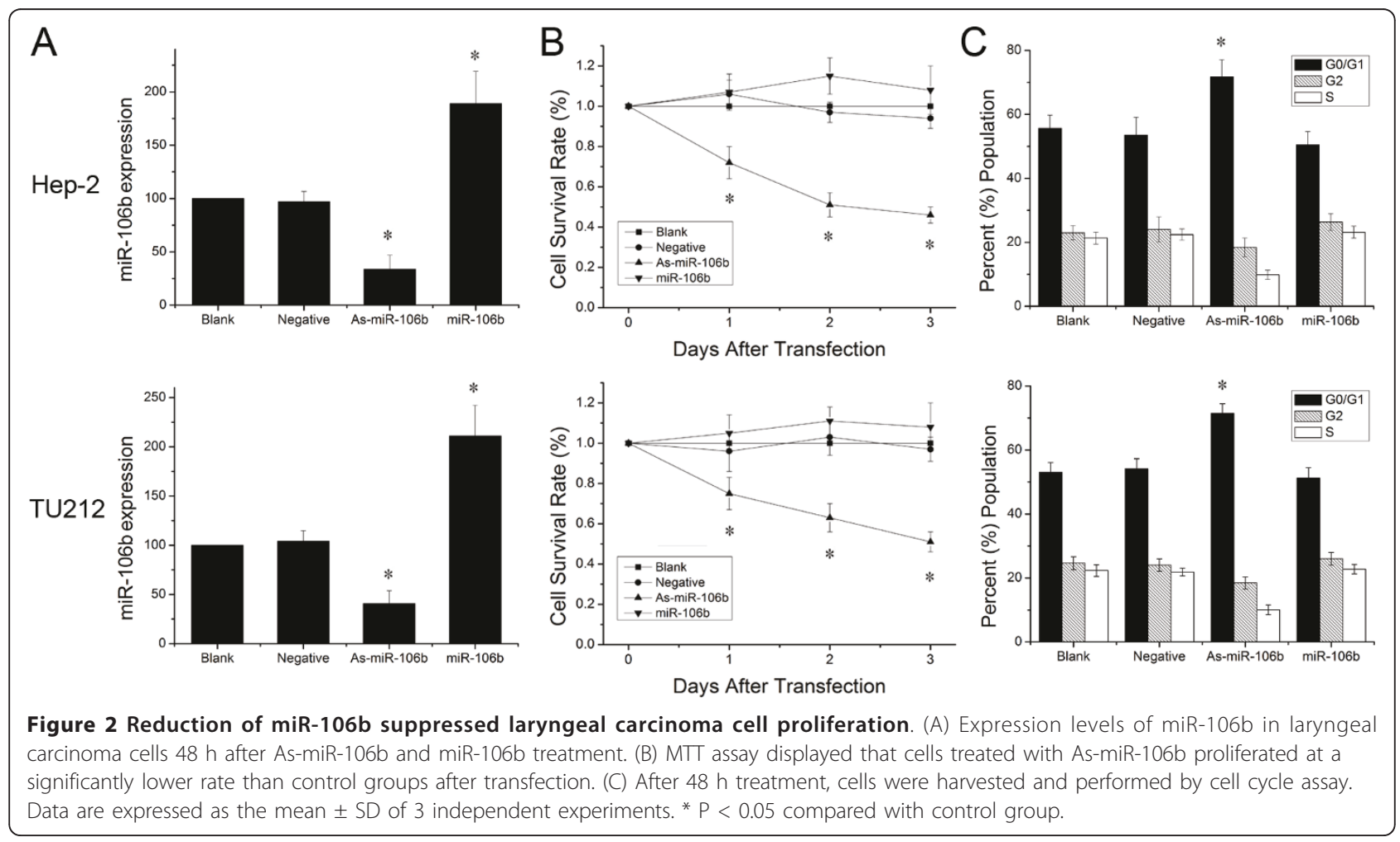




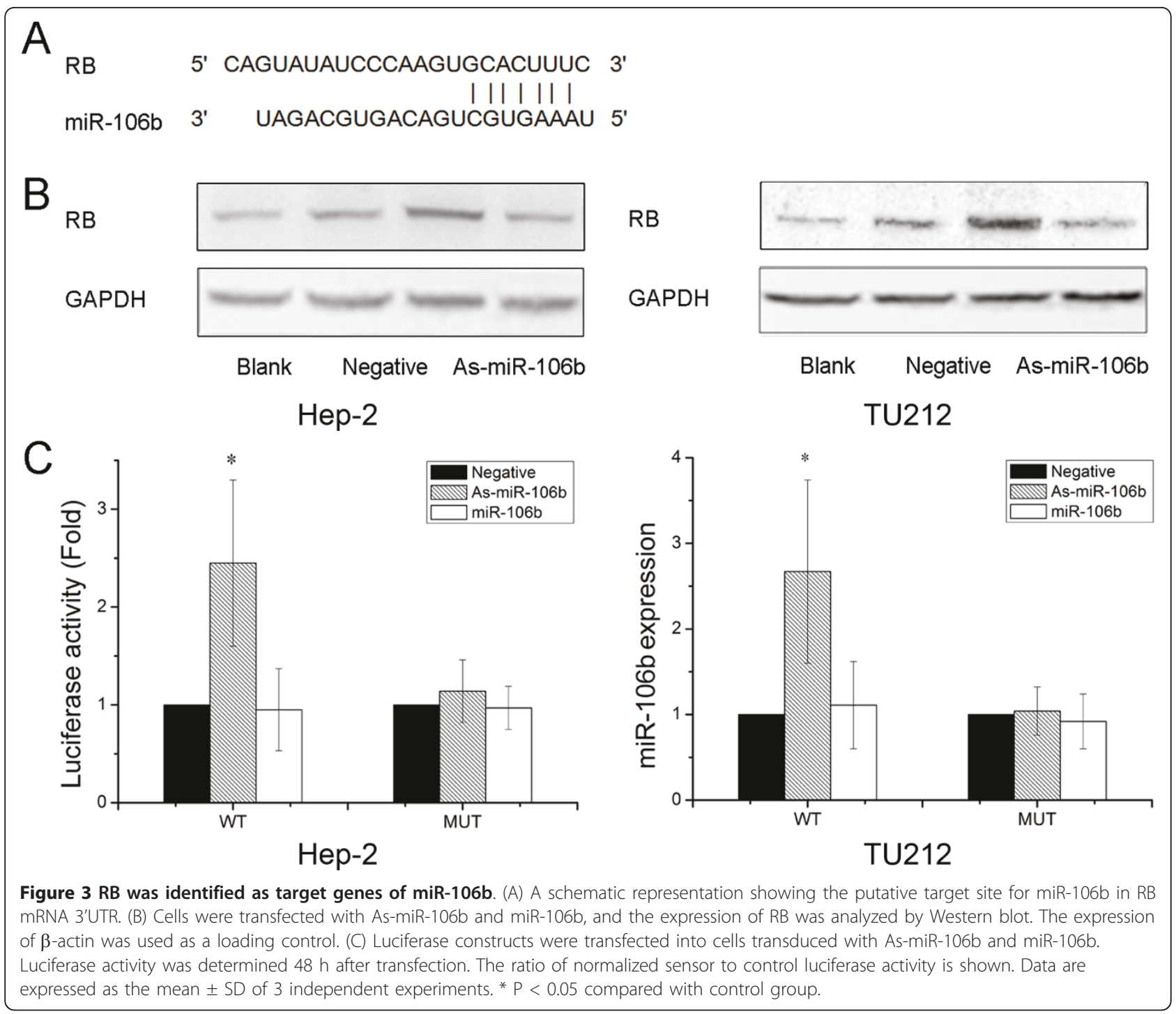

increase of luciferase activity of pGL3-WT-RB-3'UTR plasmid both in Hep-2 and TU212 cells, without change in luciferase activity of pGL3-MUT-RB-3'UTR (Figure $3 C)$. These data indicate that $R B$ is a direct target of miR-106b in laryngeal carcinoma.

\section{Core role of $\mathrm{RB}$ in miR-106b-mediated cell proliferation}

Having demonstrated RB as a direct target of miR-106b, we next examined the importance of RB in miR-106bmediated cell proliferation. The cell cycle distribution analysis showed that upregulation of miR-106b significantly reduced cell cycle G0/G1 phase arrest induced by serum starvation (Figure $4 \mathrm{~A}$ ). Then we transfected $\mathrm{Rb}$ without 3'UTR into Hep-2 cells. Western blot assay showed that transfection with RB without 3'UTR overrided $R B$ expression targeted by miR-106b (Figure 4B). As shown in Figure $4 \mathrm{C}$, the cells transfected RB significantly induced G0/G1 phase arrest. However, when we transfected with RB without 3'UTR and miR106b, expression of RB largely abrogated the effect of miR-106b on cell cycle distribution. These findings suggest that $R B$ is a major target of miR-106b involved in laryngeal carcinoma cell proliferation.

Inverse correlation of expression of miR-106b and RB in laryngeal carcinoma tissues

We further explored the correlation of between miR$106 \mathrm{~b}$ and RB expression in laryngeal carcinomas. We tested RB expression in these 20 human laryngeal carcinoma specimens and found RB expression was downregulated in laryngeal carcinomas with stage III and IV in comparison to those with stage I and II (Figure 5A). Further, Pearson correlation showed that a significant negative correlation existed between miR-106b and RB 


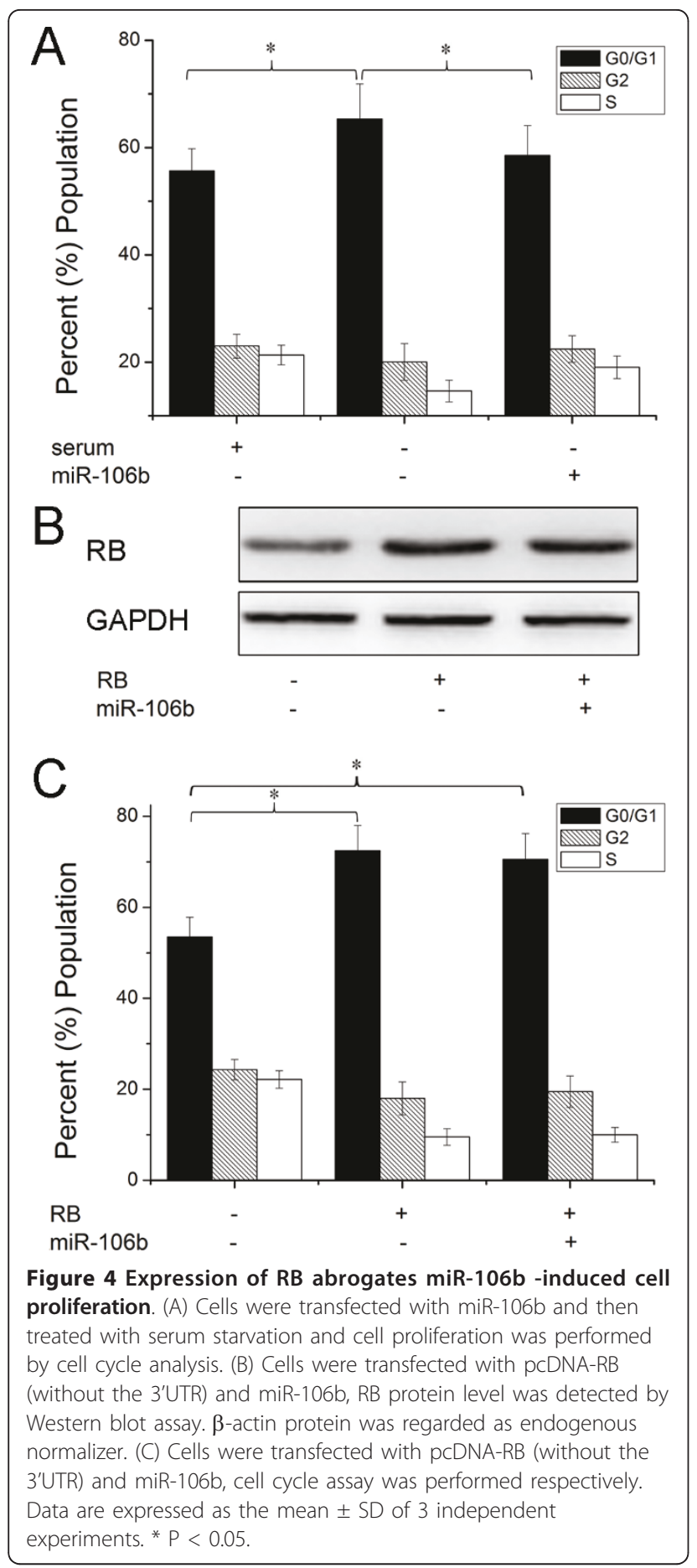

expression in laryngeal carcinoma tissues $(\mathrm{R}=0.673, \mathrm{P}$ $<0.005$ ) (Figure 5B).

\section{Discussion}

Recent evidences indicate that miR-106b has participated in development and progression of human tumors, such as hepatocellular cancer, prostate cancer, gastric cancers and renal cell carcinoma [7-10]. In this study, repression of miR-106b resulted in cell proliferation inhibition and cell cycle G0/G1 arrest in laryngeal carcinoma cells. Further, As-miR-106b regulated RB expression via targeting 3'UTR of RB. Finally, expression of RB abolished cell proliferation of miR-106b.

MiR-106b, located at Chr 7, is one member of miR106b-25 cluster. Several genes have been evidenced to be the targets of miR-106b, such as p21/CDKN1A and TGF- $\beta$ type II receptor (T $\beta$ R II). Ivanovska et al reported that miR-106b gain of function promotes cell cycle progression, whereas loss of function reverses this phenotype. And p21/CDKN1A is a direct target of miR$106 \mathrm{~b}$ and that its silencing plays a key role in miR-106binduced cell cycle phenotypes [11]. In the pathogenesis of Alzheimer's diseases, miR-106b regulated T $\beta \mathrm{R}$ II expression via binding 3' UTR of the T $\beta$ R II mRNA, thereby leads to impairment in TGF- $\beta$ signaling [12]. Here, we evidenced that RB was a novel direct and functional target of miR-106b involved in cell proliferation of laryngeal carcinoma cells. Reduction of miR-106b regulated RB expression via targeting 3'UTR of RB, and expression of RB largely abrogated miR-106b-induced cell proliferation in laryngeal carcinoma cells. And miR$106 \mathrm{~b}$ increased with the increasing stages of laryngeal carcinoma tissues, and inversely correlated with RB expression.

The RB-pathway, consisting of inhibitors and activators of cyclin-dependent kinases, the retinoblastoma tumor suppressor (RB), the E2F-family of transcription factors and cyclin-dependent protein kinases, plays critical roles in the regulation of cell cycle progression and cell death $[13,14]$. Components of this pathway, particularly RB, p16Ink4a, and cyclin D1, are frequently altered in human cancers to promote deregulated cellular proliferation $[15,16]$. Recently, a comprehensive analysis of the genome and transcriptome has shown that the RBpathway is altered in $78 \%$ of the primary glioblastoma tumor samples [17]. In our study, RB was lower expression in laryngeal carcinomas with stage III and IV in comparison to those with stage I and II, in line with the previous study [18]. And upregulation of RB controls G1/S transition in the cell cycle. Up to now, the approaches that specifically target the RB-pathway have been used in preclinical models, but not yet in the clinical setting $[19,20]$. However, the RB-pathway is still a promising target in cancer intervention and further investigations are needed.

In conclusion, we have showed that miR-106b is one of oncogenic miRNAs in laryngeal carcinomas and RB is a novel and critical target of miR-106b. These results suggest that miR-106b might be useful as a potential therapeutic target for laryngeal carcinoma and more in depth analysis is required. 

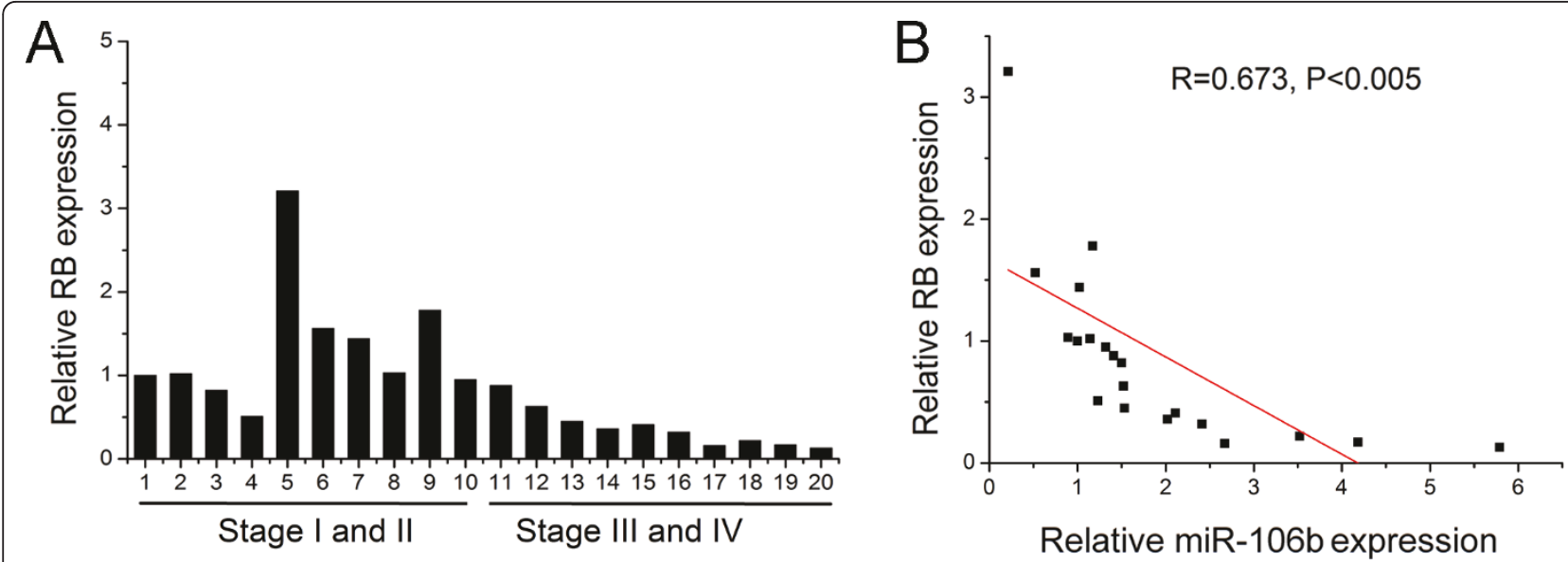

Figure 5 MiR-106b inversely correlates with RB expression in laryngeal carcinoma tissues. (A) Expression levels of RB in laryngeal carcinoma tissues were measured by Real time PCR and quantified as described in methods. (B) Inverse correlation of miR-106b expression with RB expression in laryngeal carcinoma tissues by Pearson correlation analysis. Data are presented as the means of triplicate experiments.

\section{Acknowledgements}

This work was supported by grant which is funded by Taizhou People's Hospital for the construction of Jiangsu province hospital clinical key subjects.

\section{Authors' contributions}

CK have made substantial contributions to acquisition of data. WY participated in the design of the study and performed the statistical analysis. BX participated in its design and drafted the manuscript. All authors read and approved the final manuscript.

\section{Competing interests}

The authors declare that they have no competing interests.

Received: 31 May 2011 Accepted: 8 August 2011

Published: 8 August 2011

\section{References}

1. Marioni G, Marchese-Ragona R, Cartei G, Marchese F, Staffieri A: Current opinion in diagnosis and treatment of laryngeal carcinoma. Cancer Treat Rev 2006, 32:504-515.

2. Papadas TA, Alexopoulos EC, Mallis A, Jelastopulu E, Mastronikolis NS, Goumas P: Survival after laryngectomy: a review of 133 patients with laryngeal carcinoma. Eur Arch Otorhinolaryngol 2010, 267:1095-1101.

3. Shi L, Cheng Z, Zhang J, Li R, Zhao P, Fu Z, You Y: hsa-mir-181a and hsamir-181b function as tumor suppressors in human glioma cells. Brain Res 2008, 1236:185-193.

4. Huang K, Zhang JX, Han L, You YP, Jiang T, Pu PY, Kang CS: MicroRNA roles in beta-catenin pathway. Mol Cancer 2010, 9:252.

5. Long XB, Sun GB, Hu S, Liang GT, Wang N, Zhang XH, Cao PP, Zhen HT, Cui YH, Liu Z: Let-7a microRNA functions as a potential tumor suppressor in human laryngeal cancer. Oncol Rep 2009, 22:1189-1195.

6. Hui AB, Lenarduzzi M, Krushel T, Waldron L, Pintilie M, Shi W, PerezOrdonez B, Jurisica I, O'Sullivan B, Waldron J, et al: Comprehensive MicroRNA profiling for head and neck squamous cell carcinomas. Clin Cancer Res 2010, 16:1129-1139.

7. Li Y, Tan W, Neo TW, Aung MO, Wasser S, Lim SG, Tan TM: Role of the miR-106b-25 microRNA cluster in hepatocellular carcinoma. Cancer Sci 2009, 100:1234-1242

8. Li B, Shi XB, Nori D, Chao CK, Chen AM, Valicenti R, White Rde V: Downregulation of microRNA 106b is involved in p21-mediated cell cycle arrest in response to radiation in prostate cancer cells. Prostate 2011, 71:567-574.

9. Tsujiura M, Ichikawa D, Komatsu S, Shiozaki A, Takeshita H, Kosuga T, Konishi H, Morimura R, Deguchi K, Fujiwara H, et al: Circulating microRNAs in plasma of patients with gastric cancers. Br J Cancer 2010, 102:1174-1179.

10. Slaby O, Jancovicova J, Lakomy R, Svoboda M, Poprach A, Fabian P, Kren L, Michalek J, Vyzula R: Expression of miRNA-106b in conventional renal cell carcinoma is a potential marker for prediction of early metastasis after nephrectomy. J Exp Clin Cancer Res 2010, 29:90.

11. Ivanovska I, Ball AS, Diaz RL, Magnus JF, Kibukawa M, Schelter JM, Kobayashi SV, Lim L, Burchard J, Jackson AL, et al: MicroRNAs in the miR$106 \mathrm{~b}$ family regulate $\mathrm{p} 21 / \mathrm{CDKN} 1 \mathrm{~A}$ and promote cell cycle progression. $\mathrm{Mol}$ Cell Biol 2008, 28:2167-2174.

12. Wang H, Liu J, Zong Y, Xu Y, Deng W, Zhu H, Liu Y, Ma C, Huang L, Zhang L, Qin C: miR-106b aberrantly expressed in a double transgenic mouse model for Alzheimer's disease targets TGF-beta type II receptor. Brain Res 2010, 1357:166-174.

13. Swiss VA, Casaccia P: Cell-context specific role of the E2F/Rb pathway in development and disease. Glia 2010, 58:377-390.

14. Du W, Searle JS: The rb pathway and cancer therapeutics. Curr Drug Targets 2009, 10:581-589.

15. Knudsen ES, Wang JY: Targeting the RB-pathway in cancer therapy. Clin Cancer Res 2010, 16:1094-1099.

16. Witkiewicz AK, Knudsen ES: RB pathway and therapeutic sensitivity: New insights in breast cancer and Tamoxifen therapy. Cell Cycle 2011, 10

17. Comprehensive genomic characterization defines human glioblastoma genes and core pathways. Nature 2008, 455:1061-1068.

18. Pietruszewska W, Klatka J, Borzecki A, Rieske P: Loss of heterozygosity for $\mathrm{Rb}$ locus and $\mathrm{pRb}$ immunostaining in laryngeal cancer: a clinicopathologic, molecular and immunohistochemical study. Folia Histochem Cytobiol 2008, 46:479-485.

19. Fry DW, Harvey PJ, Keller PR, Elliott WL, Meade M, Trachet E, Albassam M, Zheng X, Leopold WR, Pryer NK, Toogood PL: Specific inhibition of cyclindependent kinase 4/6 by PD 0332991 and associated antitumor activity in human tumor xenografts. Mol Cancer Ther 2004, 3:1427-1438.

20. Vaughn DJ, Flaherty K, Lal P, Gallagher M, O'Dwyer P, Wilner K, Chen I, Schwartz G: Treatment of growing teratoma syndrome. N Engl J Med 2009, 360:423-424

doi:10.1186/1756-9966-30-73

Cite this article as: Cai et al:: MiR-106b promotes cell proliferation via targeting RB in laryngeal carcinoma. Journal of Experimental \& Clinical Cancer Research 2011 30:73. 